\title{
Inmovilización del Miembro Inferior con Férulas de Vacío en las Urgencias extra hospitalarias
}

\author{
Lower Limb Immobilization with Vacuum Splints in \\ out-hospital emergency
}

\author{
Eva Compés Molina ${ }^{1}$, Cristina Pérez Díaz ${ }^{2}$, Sara Ríos Alonso ${ }^{3}$, \\ Lorena SÁnchez-CANo Burgueño ${ }^{4}$
}

\author{
${ }^{1}$ Diplomado en Enfermería y Diplomado en Fisioterapia. SAR Navalcarnero (Área 8), Madrid. \\ ${ }^{2}$ Diplomado en Enfermería. Servicio de Urgencia. Hospital del Tajo, Aranjuez. Madrid. \\ ${ }^{3}$ Diplomado en Enfermería. Servicio Hospitalización. Hospital Universitario Fundación Alcorcón. Madrid. \\ ${ }^{4}$ Diplomado en Enfermería. Servicio de Hospitalización. Hospital Universitario de Getafe.
}

\author{
Correspondencia: \\ Eva Compés Molina Navalcarnero \\ SAR Navalcarnero (Área 8), \\ C/ La Doctora, 10. Navalcarnero \\ Madrid
}

Fecha de recepción: 17 de febrero de 2011

Fecha de aceptación: 21 de marzo de 2012

Los autores declaran no tener ningún tipo de interés económico o comercial.

\section{RESUMEN}

En la práctica diaria de las emergencias extrahospitalarias y atención urgente, nos podemos encontrar con situaciones de riesgo vital extremo, situaciones en las cuales la formación del personal sanitario así como las destrezas y conocimientos sobre utilización del material de rescate, van a determinar la evolución de las lesiones. Es por tanto nuestro objetivo, aportar mayor información de la que se conoce actualmente, sobre la técnica de fijación o inmovilización de fracturas. El tratamiento de estas, en la mayoría de los casos, va a depender de las características de la lesión aunque un factor común a todas las circunstancias es la atención y manejo inicial, es decir, la inmovilización y estabilización de las lesiones con el material adecuado y con el objetivo de disminuir riesgos de alteración en otras estructuras anatómicas como consecuencia de la fractura. Esto, lo podemos conseguir con la utilización de inmovilizadores con vacío.

Palabras clave: férula; vacío; dolor; emergencia; atención urgente.

\section{ABSTRACT}

Every day the practice of hospital emergency and urgent care, we can find extreme life-threatening situations, situations in which the training of health personnel and the skills and knowledge on use of rescue material, will determine the evolution of injuries. It is therefore our aim is to provide more information than is currently known about the technique of fixation or immobilization of fractures. The treatment of these, in most cases, will depend on the characteristics of the injury although facts that are common to all the circumstances is the initial care and management, namely, immobilization and stabilization of the lesions with the appropriate material and in order to reduce risk of alteration in other anatomical structures as a result of the fracture. This, we can achieve with the use of vacuum immobilizers or vacuum cast.

Key words: cast/splint; vacuum; pain; emergency; urgent care.

Sumario: 1. Introducción. 2. Descripción de la técnica. 3. Discusión. 4. Conlusión. Bibliografía.

Referencia normalizada: Compés Molina, E., Pérez Díaz, C., Ríos Alonso, S., Sánchez-Cano Burgueño, L. Inmovilación del Miembro Inferior con Férulas de vacío en las Urgencias extra hospitalarias. Rev. Int. Cienc. Podol. 2013; 7(2): 71-76. 


\section{INTRODUCCIÓN}

En la asistencia extra hospitalaria el manejo de los pacientes con traumatismo de extremidades es distinto al que se puede ofrecer a nivel hospitalario. Para ello es preciso mantener el miembro en las mejores condiciones posibles hasta el tratamiento en el hospital. El objetivo es conseguir un alineamiento de todas las estructuras del miembro afectado para recuperar la morfología normal del mismo, y no una reducción anatómica de la fractura, pues ésta debe ser realizada posteriormente en el hospital una vez que se sepa el diagnóstico radiológico definitivo ${ }^{1}$.

En la atención extra hospitalaria de las lesiones que nos ocupan, una vez estabilizado el paciente desde el punto de vista vital, se procura el mantenimiento del segmento o articulación afectado en las mejores condiciones posibles mediante distintos métodos de inmovilización, hasta que se instaure en el hospital un diagnóstico y tratamiento definitivos. Así, se consigue minimizar las lesiones vasculares y nerviosas secundarias que se pueden asociar al traumatismo y que pueden dejar secuelas, además de reducir considerablemente el dolor del paciente durante el traslado al hospital. Queda, por tanto, de manifiesto, que la atención de las lesiones en las extremidades en la primera fase de la asistencia al paciente politraumatizado queda en un segundo plano, ya que raramente son una amenaza para la vida del paciente ${ }^{2}$.

En esta fase, normalmente se tiende a buscar inmediatamente una lesión en las estructuras óseas, pudiendo estar afectados además los músculos que circundan al hueso lesionado, y lo que sería más comprometedor, los vasos sanguíneos o los nervios que se encuentran en la vecindad de la fractura, siendo esto último lo que más influiría en la propia priorización de la lesión y en el pronóstico de la misma².

Las fracturas se definen como una rotura total o parcial en la continuidad del hueso. La rotura puede estar causada por la aplicación de una fuerza directa e importante sobre el hueso, por una fuerza indirecta a un nivel distinto del foco de fractura, por contracturas musculares potentes, por estrés o fatiga o producirse como consecuencia de la aplicación diaria de una fuerza sobre un hueso que ha sido debilitado por una patología preexistente, en cuyo caso hablamos de fractura patológica.

Ante un paciente con una fractura debemos valorar:

- Historia de la lesión; cómo se produjo, capacidad de movimiento, antecedentes de enfermedad o cirugía previa.

- Síntomas en partes blandas tales como dolor, hemorragia visible o no, edemas, espasmos musculares, etc.

- Síntomas en huesos; pérdida del contorno normal, luxación o movilidad anormal de la articulación, crepitación, uso limitado, actitud de defensa del área lesionada, etc.

- La actitud inicial será inmovilizar precozmente, aplicar hielo, elevar el miembro y colocar férula. Si se trata de una fractura abierta, realizaremos una limpieza con suero fisiológico y antisépticos, cubriendo posteriormente de forma al menos aséptica y en la medida de lo posible estéril, con el fin de evitar complicaciones secundarias.

- Es importante averiguar si está inmunizado contra el tétanos y dar antibióticos y fármacos prescritos para tratar principalmente el dolor ocasionado por la fractura (analgesia).

- Los objetivos del tratamiento son los siguientes:

- Reducción de la fractura, se realizará bajo sedación del paciente.

- Inmovilización mediante férulas, vendajes funcionales, yeso, tracciones, fijación interna o externa.

- Restauración de la función o rehabilitación.

Las fracturas se pueden clasificar según distintos aspectos (Tabla 1$)^{2}$.

Una vez alineada la extremidad fracturada, se procede a su inmovilización con métodos provisionales durante la estabilización y traslado del herido, hasta su inmovilización definitiva en el centro hospitalario adecuado. De esta forma los fragmentos óseos permanecen alineados, disminuyendo el dolor y la iatrogenia que pudiera producirse durante la movilización y transporte del paciente. En los servicios de emergencias la inmovilización se realiza con férulas ${ }^{2}$.

Férula: Tablilla flexible y resistente que se emplea en el tratamiento de las fracturas ${ }^{3}$. 
Tabla 1. Clasificacion de las fracturas.

\begin{tabular}{l|l}
\hline Según su imagen radiográfica & Según la afectación de tejidos blandos \\
\hline $\begin{array}{l}\text { 1. Transversal } \\
\text { 2. Oblicua }\end{array}$ & 1. Cerradas \\
3. Espiral & 2. Abiertas \\
4. Conminuta & \\
5. Avulsión & \\
6. Impactada & \\
7. Abultadas & \\
8. En tallo verde &
\end{tabular}

En el caso de la férula de vacío, diremos que su peculiaridad es que realiza su función mediante la aplicación de vacío a una funda cuyo interior está relleno de pequeñas partículas de poliéster, que se acoplan perfectamente al miembro a inmovilizar. Actualmente se considera que la inmovilización que se consigue por este método es mejor y más fiable que la obtenida mediante férulas neumáticas. Su efecto es el contrario que las anteriores, es decir, hacemos el vacío mediante una bomba y al sacar el aire, estas partículas se quedan completamente unidas y compactas sobre el miembro afectado, quedando este completamente inmovilizado. Cabe destacar que son permeables a los rayos $\mathrm{X}$, lo cual facilita el estudio radiológico del miembro afectado, sin necesidad de retirar la férula. También tienen la ventaja de comprimir puntos sangrantes en los miembros afectos ${ }^{1,6}$.

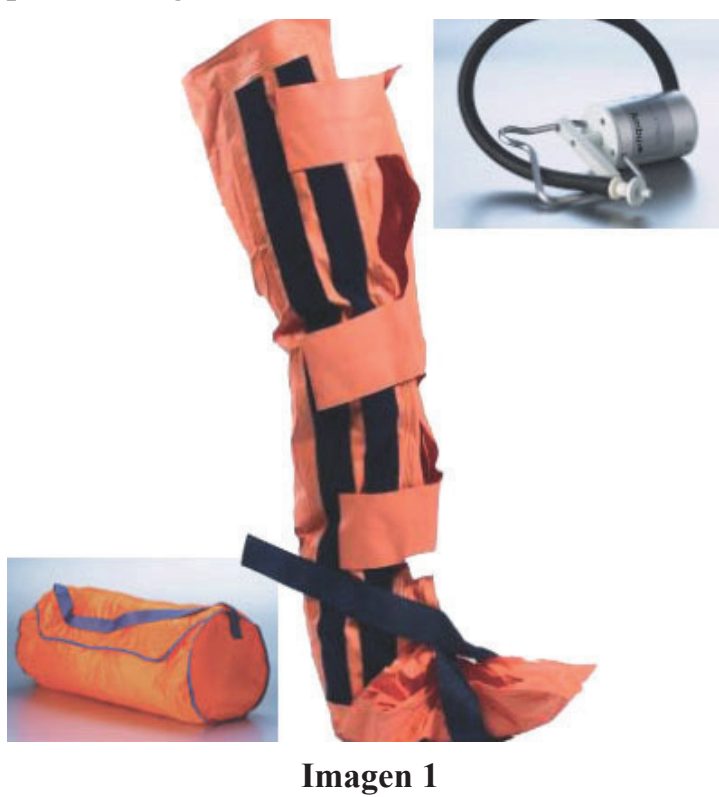

En general, las férulas abarcarán la articulación distal y proximal con respecto al foco de fractura para garantizar una buena inmovilización.

Existen varios tipos de férulas para miembros: - Rígidas: deformables y no deformables.

- Flexibles: neumáticas y de vacío.

- De tracción.

La férula de vacío es de primera elección para el traslado de cualquier paciente herido con fracturas de extremidades inferiores o superiores en las urgencias extra hospitalarias. Esto es así por su capacidad de inmovilizar, amortiguar las vibraciones, ser menos iatrogénica y más confortable para el paciente ${ }^{2}$.

La fácil colocación de la misma hace que sea especialmente eficaz, permitiendo una correcta inmovilización, evitando lesiones secundarias (musculares, daño vascular, lesiones nerviosas, etc.) y disminuyendo el dolor, por lo que facilitan el traslado del herido.

Tienen la misma técnica de colocación y formas anatómicas que las férulas hinchables, salvo que el sistema de cierre suele ser tipo "velcro", y se puede ocasionar en algunos casos, una mayor movilización del miembro al cerrar la férula (Imagen 1) ${ }^{4}$.

En los traslados aéreos se debe tener en cuenta que, al disminuir la presión atmosférica con la altitud, pueden perder consistencia y, por tanto, no inmovilizar de forma suficiente ${ }^{5}$.

\section{DESCRIPCIÓN DE LA TÉCNICA}

El objetivo de las férulas es prevenir el movimiento en las puntas óseas fracturadas, lo que 
disminuye la incidencia de dolor, discapacidad y complicaciones graves. También elimina el daño a los músculos, nervios y vasos sanguíneos.

La aplicación de férulas debe emplearse para pacientes estables. En pacientes politraumatizados inestables, es mejor realizar la inmovilización espinal completa para evitar la pérdida de tiempo en la escena.

\section{Técnica de colocación}

Al tomar la decisión de colocar una férula en una extremidad debe tenerse en cuenta:

- Revisar previamente la integridad de la férula y el buen funcionamiento de la bomba de vacío (pues puede estar rasgado y no realizar el vacío necesario).

- Antes de su utilización, se debe quitar la ropa que pueda apretar bajo la férula.

- Se deben cubrir las heridas abiertas con apósitos estériles.

- Se procede, aplicar una ligera tracción para alinear la extremidad.

- Valorar pulsos periféricos (pedio, tibial posterior y poplíteo).

- Dejar la férula de vacío plana sobre todas las correas abiertas y con la superficie interna de cara hacia arriba.

- Alisar uniformemente las bolitas de poliespam de toda la férula para garantizar una distribución uniforme.

- Se debe sujetar el miembro por encima y por debajo de la zona de lesión al colocar la férula alrededor de la extremidad. Debe inmovilizarse tanto la articulación superior como la inferior respecto a la zona de la lesión.

- Dar forma a la férula en los lados y la parte superior de la extremidad.

- Fijar la férula con las correas de velcro.

- Doblar la parte distal de la férula hacia fuera si es necesario para poder inspeccionar los dedos de los pies.

- Evacuar el aire hasta que la férula pase de ser un dispositivo blando y maleable a tener una rigidez similar al yeso.

- Revisar que se mantiene el vacío, comprobando la rigidez de la férula.

- Reevaluar constantemente la presencia de pulsos pedios, coloración y signos de buena perfusión en la zona de la lesión, antes y durante el traslado del paciente al centro hospitalario.

\section{Técnica de retirada}

- Para retirar la férula, abrir la válvula para eliminar el sistema de vacío, paulatinamente.

- Cuando la férula sea maleable, abrir las correas y sostener cuidadosamente la extremidad para quitar la férula.

- Mantener inmovilización ${ }^{5,6}$.

\section{DISCUSIÓN}

El uso de las férulas de vacío es relativamente reciente, pero ha demostrado ser uno de los métodos más eficaces de estabilización de fracturas para su traslado al centro sanitario (Imagen 2$)^{7}$.

Si bien, presenta algunos inconvenientes en su uso extrahospitalario, cabe destacar que sus ventajas son considerables. Entre ellas destacamos la solidez y resistencia del material al roce con superficies erosivas, que lo hacen más aconsejable en este medio en el que no es infrecuente la posibilidad de tener que utilizarlas sobre terrenos arenosos o cortantes. No son totalmente irrompibles, pero sí claramente más resistentes que las neumáticas ${ }^{8}$.

Es importante, además, que una vez colocadas, ocupan menos espacio que las hinchables

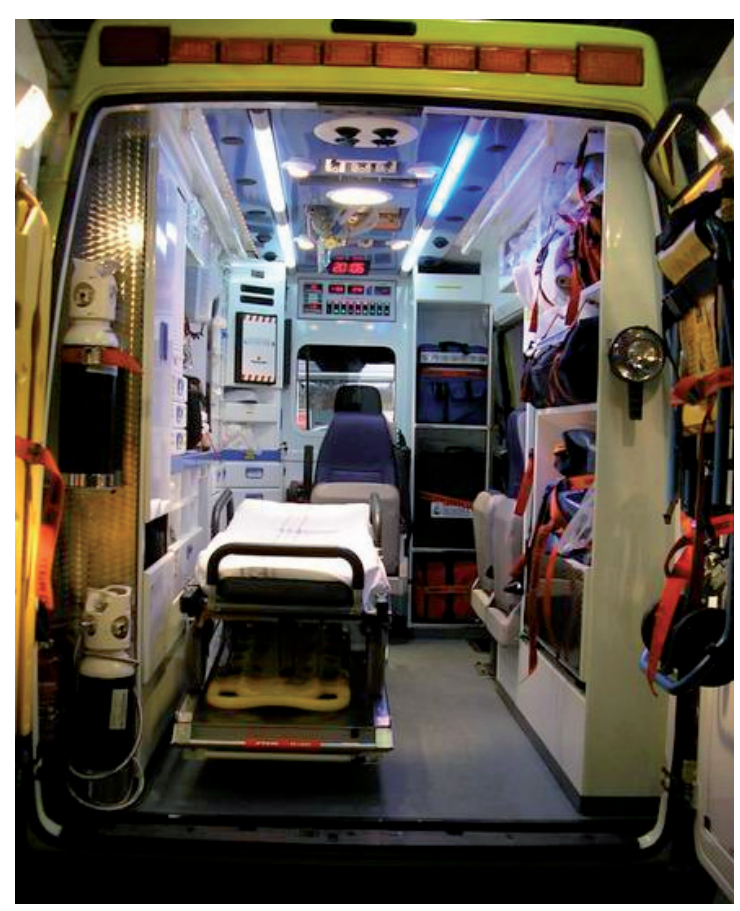

Imagen 2 
y, por tanto, pueden ser más cómodas en extricaciones complejas.

La única ventaja de la que hemos encontrado reseñas debidamente estudiadas, es aquella que se refiere a la comodidad referida por el usuario. Recibe una valoración bastante superior en su uso extra e intrahospitalario (inmovilizaciones para pruebas diagnósticas y radiológicas, sobre todo $)^{9,10}$.

Otra ventaja que encontramos en este tipo de férulas, poniéndolas en comparación con las rígidas, es el espacio que ocupan en la ambulancia. Al ser plegables, son fácilmente almacenables en su bolsa y ocupan poco espacio, siendo éste tan importante en el reducido habitáculo del vehículo ${ }^{8}$.

En lo referente a los inconvenientes, diremos que hemos encontrado que uno de los más importantes es su peso, que es más elevado que el de las férulas neumáticas, y por tanto son más incómodas de transportar hasta el herido. A esto hay que añadir que es necesario para su correcto uso, una bomba de vacío, sin la cual no obtendremos ninguna utilidad de la férula. Esto supone que haya que evaluar su correcto funcionamiento, la integridad de las válvulas y su limpieza, etc. ${ }^{11}$

Otro aspecto en su contra es su coste económico, ya que supone más del doble de coste que las anteriormente señaladas, aunque es de más difícil valoración puesto que ya hemos expuesto que este tipo de férulas son más resistentes y, por lo tanto duran más, si bien no hemos encontrado información alguna relativa a este aspecto $^{12,13}$.

\section{CONCLUSIÓN}

Nos hemos encontrado con serias dificultades a la hora de encontrar información relativa a este tipo de férulas y que no estuviese únicamente referida a su modo de empleo, indicaciones, etc. Creemos que no existen estudios suficientes referidos a aspectos relativos a la relación coste-efectividad, comodidad en el uso por parte del personal sanitario o idoneidad para el transporte del paciente poli traumatizado que requiera este tipo de atención. Tan sólo podemos hacer referencia a estudios que se refieren a la comodidad percibida por el usuario.

\section{BIBLIOGRAFÍA}

1. Monfort Masiá A. Manejo del paciente con fractura del miembro superior e inferior. Enferm. Integra.2004; $67: 9-15$

2. Mañanes González E, López Sánchez MT. Traumatismos de extremidades En: Fernández Ayuso D, Aparicio Santos J, Pérez Olmo JL,Serrano Moraza A. Manual de Enfermería en Emergencia Pre hospitalaria y Rescate. $2^{\circ}$ edición. Madrid: 2002; Aran Ediciones S.L.

3. Diccionario de la Real Academia de la Lengua Española. [acceso 2 junio 2010]. Disponible en www. rae.es

4. Casal C, Carmona JV. Tratado de Cuidados Críticos Pediátricos y neonatales [monografía en internet] España. Grupo de Enfermería de Cuidados Críticos Pediátricos y Neonatales; 2006 [acceso 2 junio 2010]. Disponible en www.eccpn.albarra.org

5. Briebe del Río P. Manual de trasporte y movilización del paciente crítico. 2003. Madrid. Ed. Formación de Alcalá.

6. Proehl JA. Enfermería de urgencias. Tecnicas y procedimientos, 2005. Madrid; Elsevier.

7. Hartwellmedical.com [sede Web]. EEUU. Hartwells Medical; 1999 [actualizado 10 septiembre 2009; acceso 10 junio 2010]. FAQ; [aproximadamente 3 pantallas]. Disponible en http://www.hartwellmedical.com/faqevac.html

8. Hartwellmedical.com [sede Web]. EEUU. Hartwells Medical; 1999 [actualizado 10 septiembre 2009; acceso 10 junio 2010]. FAQ; [aproximadamente 6 pantallas]. Disponible en http://www.hartwellmedical.com/testimonials.html

9. Bale RJ, Vogele M, Rieger M, BuChbergeri W, Lukas P, JasChke W.Technical Innovation: A new vacuum device for extremity immobilization. AJR. 1999; 172, April.

10. Letts RM, Hobson DA. The vacuum splint: an aid in emergency splinting of fractures. Can Med Assoc 1973 Oct 6;109(7):599-600. 
11. Hartwellmedical.com [sede Web]. EEUU. Hartwells Medical; 1999 [actualizado 10 septiembre 2009; acceso 10 junio 2010]. FAQ;[aproximadamente 3 pantallas]. Disponible en http://www.hartwellmedical.com/evac.html

12. Lifemedicalsupplier.com [sede Web]. Florida. Life Medical Supplier; 2010 [acceso 11 junio 2010]; Inmobilization, extrication, emergency vacuum splints [aproximadamente 2 pantallas]. Disponible en:http://www.lifemedicalsupplier.com/inmovilizacion-y-extricacion-inmovilizadores-al-vacioc-16_24.html

13. Quadmed.com [sede Web]; EEUU. 2010 [acceso 20 junio 2010]; www.quadmed.com/splints-c95.html [aproximadamente 2 pantallas]. Disponible en: www.quadmed.com 